\title{
Baclofen as adjuvant to weaning from mechanical ventilation in a patient with intractable hiccup. A Case Report
}

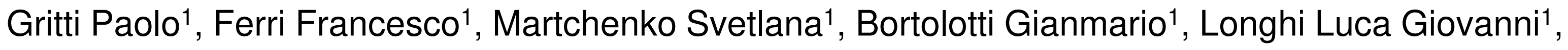 \\ Lorini Ferdinando Luca ${ }^{1}$
}

1 ASST Papa Giovanni XXIII Bergamo, Dept of Anaesthesiology \& Intensive Care, Bergamo, Italy

\section{BACKGROUND}

Lateral medullary syndrome (LMS), better known as Wallenberg syndrome is a pattern of neurologic symptoms due to the tissue ischemia and necrosis of the lateral part of the medulla in the brain. This syndrome was originally described in 1895 , and was characterized by sensory deficits affecting the trunk and extremities on the opposite side of the infarction and sensory deficits affecting the face and cranial nerves on the same side. ${ }^{1}$ Rarely Wallenberg syndrome can result in violent and incoercible hiccups. ${ }^{2}$ Here we report a case of a patient with a LMS and intractable hiccups who was unweaning by mechanical ventilation until high dosage of baclofen was administered.

\section{CASE REPORT}

A 50 years-old man was admitted to emergency department with an impaired sensation over left half face vertigo, nystagmus, hiccups, nausea and vomiting. He was also dysphagic, with an impaired sensation over left half face, and contralateral hemi body. Due to the magnitude of the dysphagia and hiccups the patient presented an aspiration pneumonia and as a result of respiratory failure he required intubation. A further MRI showed an acute left sided dorsal lateral medullary infarct (fig.1 A, and B). However due to violent hiccups mechanical ventilation of the patient resulted impaired, requiring continuous curare administration and impeding the weaning and extubation of the patient (fig 2, C).

The hiccups was intractable with the common metoclopramide, chlorpromazine, alprazolam, midazolam, omeprazole and gabapentin.

The patient reported resolution of hiccups 3 hours after $10 \mathrm{mg}$ oral baclofen was administrated.

\section{LEARNING POINTS}

Wallenberg syndrome can result in violent and incoercible hiccups
Figure $^{\circ} 1$. A and $B$

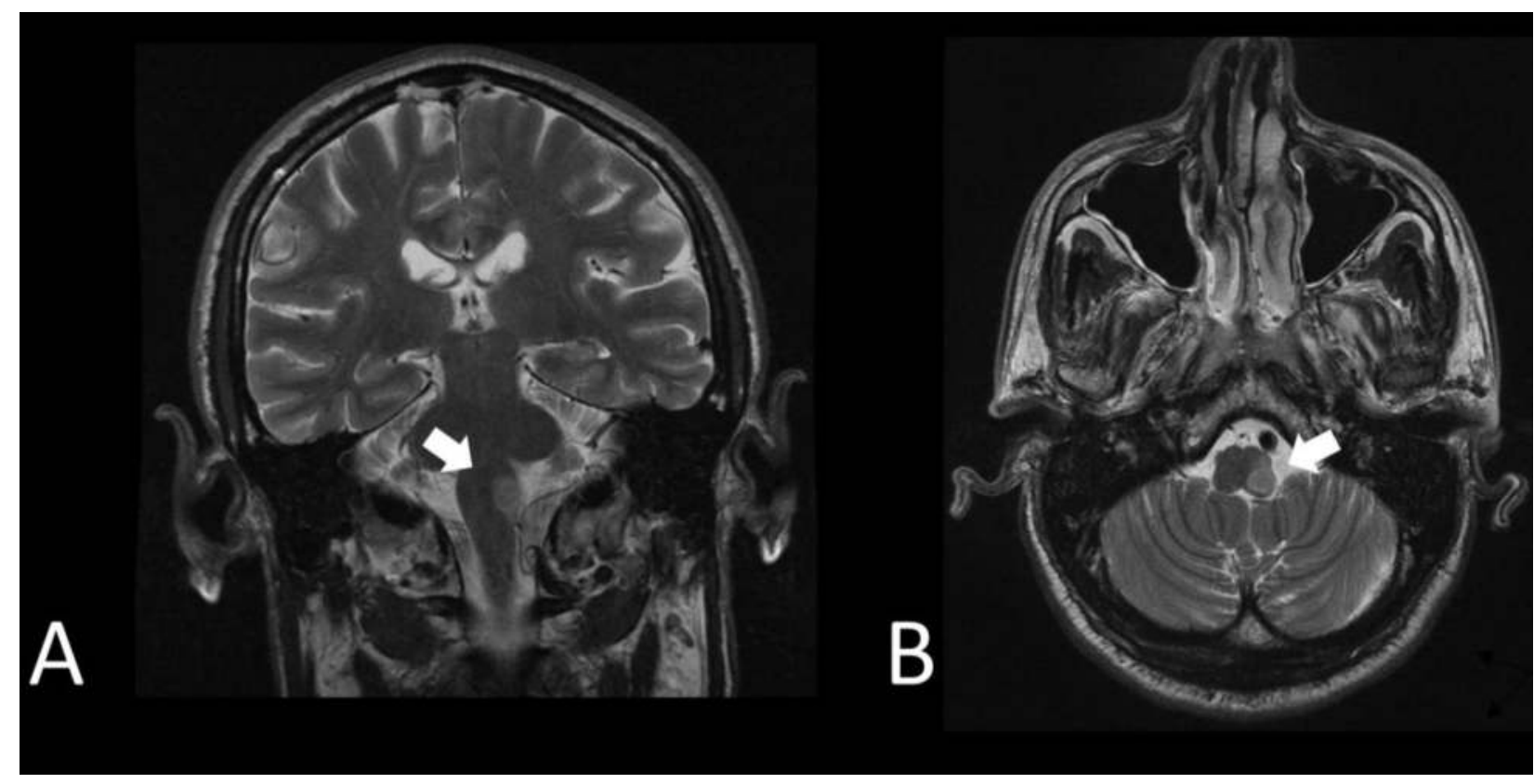

Acute left sided dorsal lateral medullary infarct (White arrow).

\section{DISCUSSION}

One of the most unusual and difficult symptoms to treat that Wallenberg syndrome have are interminable violent hiccups which involves $14 \%$ of patients. $^{2}$ It is suggested that hiccups are caused by the failure of the reciprocal inhibition of the valve control of the pharynx and larynx. ${ }^{3}$ The hiccups can be so severe that patients often struggle to eat, sleep and carry on conversations, or contribute to ab-ingestis or disadaptation to mechanical ventilation. Depending on the severity of the blockage caused by the stroke, the hiccups can last for weeks. Unfortunately there are very few successful medications available to mediate the inconvenience of constant hiccups.

Baclofen, an analog of GABA, decreases the excitability and inhibits the hiccup reflex.

\section{LEARNING POINTS}

$10 \mathrm{mg}$ Baclofen 3 times a day could be effective in treating persistent hiccups followings LMS syndrome.

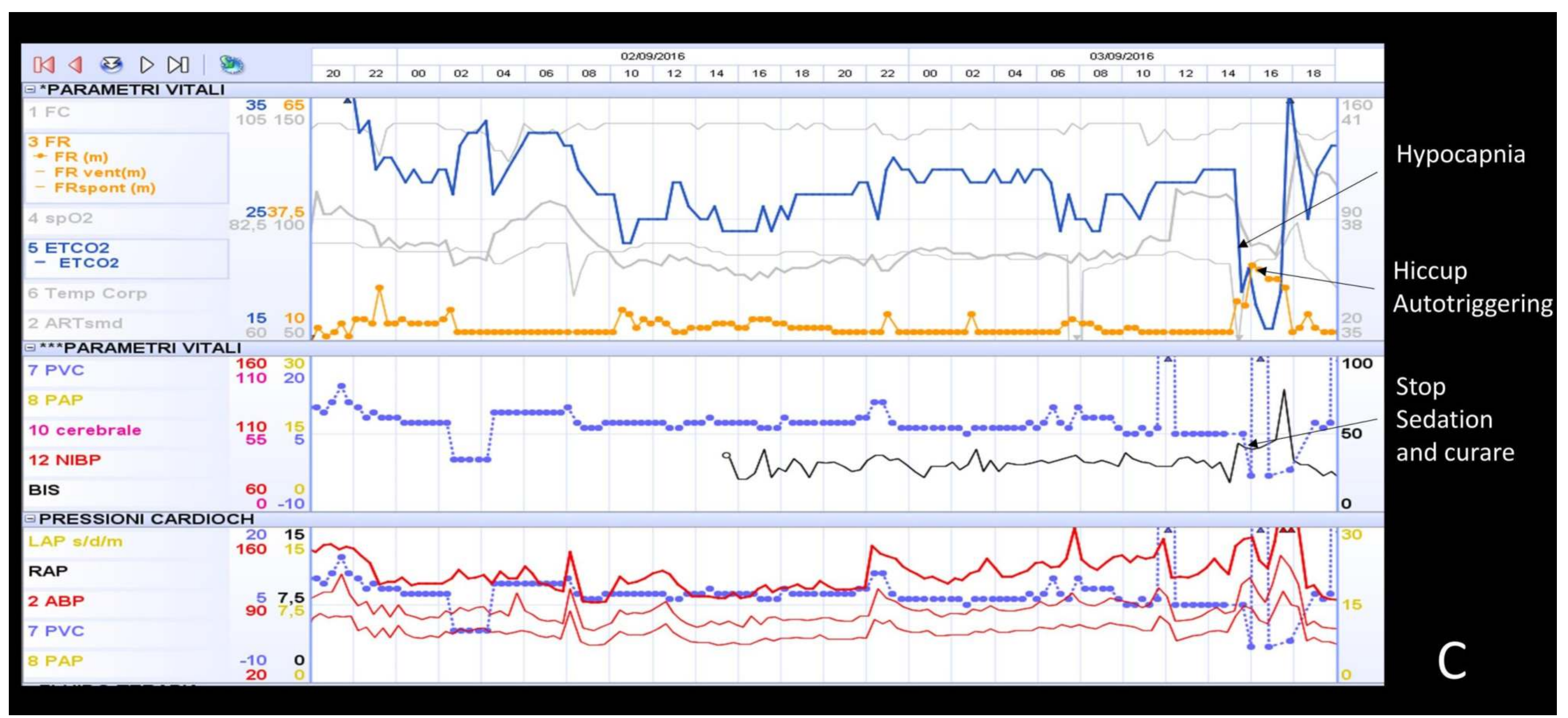

